Ann. Zootech., I974, 23 (3), 359-364.

\title{
INFLUENCE DE LA COAGULATION DES PROTÉINES DU LAIT SUR L'UTILISATION DIGESTIVE D'UN LAIT DE REMPLACEMENT PAR LE VEAU PRÉRUMINANT
}

\author{
R. TOULLEC $\left({ }^{(}\right)$, J.-F, FRANTZEN $\left({ }^{2}\right)$ et C.-M. MATHIEU $\left({ }^{1}\right)$ \\ avec la collaboration technique de Yvette Lento (1) \\ C. Marpillat et Colette Raynal \\ Station de Recherches sur l'Élevage des Ruminants, \\ Centre de Recherches de Clermont-Ferrand, I. N. R. A., \\ Theix, 63110 Beaumont
}

\section{RÉSUMÉ}

L'influence de la coagulation des protéines sur l'utilisation digestive d'un lait de remplacement (contenant $67 \mathrm{p}$. Ioo de poudre de lait écrémé par rapport à la matière sèche) a été étudiée en le rendant partiellement ou totalement incoagulable par addition de citrate de sodium ou d'acide chlorhydrique au moment de l'emploi. Les laits de remplacement ont été distribués à 3 veaux préruminants maintenus en cages à bilan entre les âges d'environ 8 et roo j. L'appétit, la croissance et l'efficacité alimentaire ont été plus satisfaisants chez les animaux qui recevaient le lait témoin et le lait citrate que chez ceux qui consommaient le lait acide (tabl. 2). La modification ou la suppression de la coagulation n'a pas eu de conséquence néfaste sur la fréquence des diarrhées. L'utilisation digestive (tabl. 3) n'a été notablement diminuée que jusqu'à l'âge de trois semaines ou d'un mois : ainsi, entre les âges d'environ I4 et $20 \mathrm{j}$, les CUD apparents de la matière organique étaient respectivement de 94,88 et $84 \mathrm{p}$. Ioo pour les laits témoin, citrate et acide au lieu de 97, 97 et $96 \mathrm{p}$. Ioo à partir de l'âge d'un mois. Cela confirme donc que l'absence de coagulation ne constitue pas un obstacle au remplacement total des protéines du lait dans les aliments d'allaitement; cependant, au cours du premier mois, il serait probablement judicieux de conserver une proportion importante de lait écrémé.

Dans les aliments d'allaitement actuellement utilisé pour l'élevage et l'engraissement du veau, une partie du lait écrémé est parfois remplacée par d'autres sources de protéines (soja, levure, poisson...). Cependant, les protéines de substitution ont généralement une utilisation diges-

Adresse actuelle :

(1) Station de Recherches zootechniques, Centre de Recherches de Rennes, 65, rue de Saint-Brieur. 35042 Rennes Cedex.

(2) Union des Coopératives agricoles d'Alimentation du Bétail, B. P. 75, o240o Château Thierry. 
tive moins élevée que celles du lait écrémé (cf. revue de Toullec et al., 1973). Cela pourrait être dû notamment à l'impossibilité, pour ces protéines, de former, comme la caséine, un coagulum ferme dans la caillette sous l'action de la présure. L'absence de coagulation peut entraîner des modifications importantes dans la digestion des aliments; ainsi, en remplaçant les protéines du lait par celles du lactosérum, nous avons observé une accélération dans la vidange stomacale des matières azotées et des matières grasses (Toullec, Thivend et Marhieu, 1971). Cependant, les conséquences de la coagulation des protéines dans la caillette sont controversées. Kastelic, Bentley et Phillips (I950) ainsi que Blaxter et Wood (I953) ont obtenu une forte augmentation de la fréquence des diarrhées chez des veaux recevant des laits de remplacement incoagulables ou dont le temps de coagulation était augmenté ; en revanche, ces effets néfastes n'ont pas été observés par Owen et al. (1958) et NetKe, Gardier et Kendall (I962).

TABLEAU I

Composition de l'aliment d'allaitement

\begin{tabular}{|c|c|c|c|}
\hline \multicolumn{4}{|c|}{ Constituants (p. 100 de l'aliment) } \\
\hline $\begin{array}{l}\text { Lait écrémé séché par le } \\
\text { procédé Spray } \ldots \ldots \ldots \\
\text { Matières grasses (i) } \ldots \ldots \\
\text { Amidon de maïs cru } \ldots \ldots\end{array}$ & $\begin{array}{r}67 \\
22 \\
3\end{array}$ & $\begin{array}{l}\text { Amidon de maïs prégéla- } \\
\text { tinisé } \ldots \ldots \ldots \ldots \ldots \ldots \\
\text { Glucose } \ldots \ldots \ldots \ldots \ldots \ldots \\
\text { Méthionine commerciale } \\
\text { Complément minéral et vi- } \\
\text { taminique } \ldots \ldots \ldots \ldots\end{array}$ & $\begin{array}{l}3 \\
4 \\
0,1 \\
0,9\end{array}$ \\
\hline
\end{tabular}

Composition chimique (p. 100 de la matière sèche)

\begin{tabular}{l|r|r|r} 
& & & \\
Matières azotées $\ldots \ldots \ldots$ & 23,4 & Substances réductrices .. & $43,4_{4}^{\prime}$ \\
Matières grasses $\ldots \ldots \ldots$ & 21,7 & Matières minérales ...... & 5,4 \\
\hline
\end{tabular}

(1) Les matières grasses étaient un mélange de suif $(73,5$ p. 100), d'huile de hareng hydrogénée $(24,5$ p. 100) et de saccharoglycérides (2 p. 100). Elles avaient été incorporées dans du lait écrémé liquide et le mélange ainsi obtenu avait été homogénéisé et séché par le procédé Spray.

Nous avons donc étudié l'influence de la coagulation sur l'état sanitaire des animaux et l'utilisation digestive des aliments. Pour cela, nous avons distribué à des veaux préruminants 3 laits de remplacement, dont l'un coagulait normalement tandis que les 2 autres avaient été rendus partiellement ou totalement incoagulables par addition de citrate de sodium ou d'acide chlorhydrique. Ces laits de remplacement ont été préparés à partir d'un même aliment d'allaitement (tabl, I) dont les protéines provenaient exclusivement du lait écrémé. Le premier (lait " témoin ") formait rapidement un coagulum ferme sous l'action de la présure. Les 2 autres (laits " citrate " et " acide ") ont été obtenus en ajoutant au moment de l'emploi au précédent, 13,3 g de citrate de sodium ou $39 \mathrm{~g}$ d'acide chlorhydrique pur (densité $\mathrm{I}, \mathrm{I} 8$ ) par $\mathrm{kg}$ de matière sèche. Le lait citrate ne coagulait plus sous l'action de la présure; il pouvait encore précipiter sous l'action de l'acide chlorhydrique, mais le coagulum ainsi obtenu était granuleux et friable. Le lait acide formait instantanément un coagulum granuleux qui était remis en suspension très 
fine par agitation énergique ; il avait alors un $\mathrm{pH}$ de 4,25 et, ni l'addition de présure, ni celle d'acide chlorhydrique ne pouvaient, in vitro, modifier son état. Ces différentes observations ont été vérifiées dans la caillette, lors de l'abattage des veaux.

Chacun des aliments a été distribué à 3 veaux mâles de race Frisonne, achetés à l'âge d'environ huit jours et placés dès leur arrivée dans des cages à bilans. Les mesures ont commencé une semaine plus tard et ont duré 87 jours comprenant une période de $6 \mathrm{j}$, 5 périodes de I I $\mathrm{j}$ et I période de $8 \mathrm{j}$, séparées par des interpériodes de $3 \mathrm{j}$. Les laits de remplacement ont été distribués au seau deux fois par jour. La concentration, qui était de rzo g d'aliment d'allaitement par $\mathrm{kg}$ de lait de remplacement au cours des deux premières périodes, augmentait progressivement pour atteindre $160 \mathrm{~g}$ à partir de la $6^{\mathrm{e}}$ période. Les quantités d'aliment d'allaitement distribuées par jour étaient importantes et augmentaient avec l'âge : elles étaient de $55 \mathrm{~g}$ par kg de poids métabolique (poids vif ${ }^{0,75}$ ) au cours des 3 premières périodes, puis de $67 \mathrm{~g}$, passant ainsi progressivement d'un minimum de I $\mathrm{kg}$ par veau à un maximum de 2,9 $\mathrm{kg}$. Les méthodes de prélèvement, de mesure, de dosage et de calcul ont été décrites précédemment (Paruelle et al., I972).

Le lait acide a été beaucoup moins bien consommé que les laits témoin et citrate, en raison probablement de son goût âpre (tabl. 2) ; l'un des veaux qui le recevaient a d'ailleurs dû être éliminé après trois semaines d'expérience à cause d'une croissance très faible et il n'en a pas été tenu compte dans l'expression des résultats. En conséquence, tout au long de l'essai, la croissance et l'efficacité alimentaire ont été beaucoup moins satisfaisantes avec le lait acide qu'avec le lait citrate et surtout le lait témoin (tabl. 2).

\section{TABLEAU 2}

Croissance, consommation et état des fèces

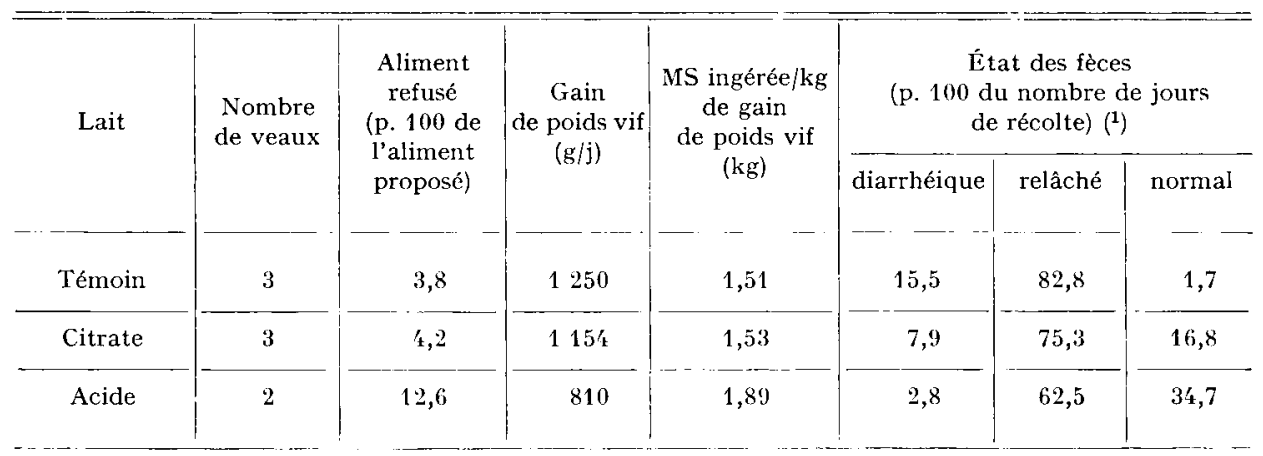

(1) L'état des fèces a été caractérisé d'après leur teneur en matière sèche : moins de 12 p. 100: état diarrhéique; entre 12 et 20 p. 100 : état relâché; plus de 20 p. 100 : état normal (BLAXTER et WooD, 1953).

La fréquence de l'état " diarrhéique "(tabl. 2) a été plus faible avec les laits citrate et acide qu'avec le lait témoin; il en a été de même pour la fréquence de l'état " relâché ". Ces résultats s'accordent avec ceux obtenus par OWEN et al. (I958) ainsi que par Burt et IRvinE (1972), avec du lait rendu incoagulable sous l'action de la présure par addition de citrate de sodium et par NeTKe et al. (1962) avec un lait dont la coagulation était retardée par addition de soude jusqu'à $\mathrm{pH}$ 8. La modification ou la suppression de la coagulation des protéines du 
lait ne semble donc pas avoir d'incidence importante sur la consistance des fèces, malgré l'accélération entraînée dans la vidange stomacale des matières azotées et des lipides par l'addition de citrate de sodium et d'acide chlorhydrique (Frantzen et al., i973).

\section{TABLEAU 3}

Utilisation digestive des laits de remplacement et rétention azotée

\begin{tabular}{|c|c|c|c|c|c|}
\hline & & & & Lait & \\
\hline & & & Témoin & Citrate & Acide \\
\hline$\frac{a^{2}}{}$ & Matière sèche & $\begin{array}{l}14-20 \\
23-34 \\
37-48 \\
51-101\end{array}$ & $\begin{array}{l}94,7 \pm 2,2 \\
97,2 \pm 0,2 \\
97,2 \pm 0,5 \\
97,1 \pm 0,6\end{array}$ & $\begin{array}{l}86,5 \pm 6,0 \\
95,7 \pm 1,8 \\
97,4 \pm 0,9 \\
96,4 \pm 0,4\end{array}$ & $\begin{array}{l}82,7 \pm 0,8 \\
92,9 \pm 0,1 \\
95,2 \pm 0,9 \\
95,6 \pm 0,9\end{array}$ \\
\hline 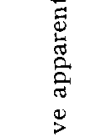 & Matière organique & $\begin{array}{l}14-20 \\
23-34 \\
37-48 \\
51-101\end{array}$ & $\begin{array}{l}94,2 \pm 3,6 \\
97,3 \pm 0,2 \\
97,3 \pm 0,4 \\
97,3 \pm 0,2\end{array}$ & $\begin{array}{l}87,6 \pm 5,3 \\
96,0 \pm 1,7 \\
97,6 \pm 0,8 \\
96,7 \pm 0,3\end{array}$ & $\begin{array}{l}84,1 \pm 1,2 \\
93,3 \pm 0,1 \\
95,5 \pm 0,6 \\
96,5 \pm 0,3\end{array}$ \\
\hline 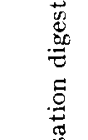 & Matières azotées & $\begin{array}{l}14-20 \\
23-34 \\
37-48 \\
51-101\end{array}$ & $\begin{array}{l}91,9 \pm 4,5 \\
95,5 \pm 0,5 \\
95,5 \pm 0,7 \\
96,2 \pm 0,5\end{array}$ & $\begin{array}{l}87,0 \pm 4,1 \\
9,3 \pm 1,8 \\
95,7 \pm 1,6 \\
95,3 \pm 0,5\end{array}$ & $\begin{array}{l}74,8 \pm 11,7 \\
89,0 \pm 1,6 \\
92,8 \pm 0,9 \\
95,6 \pm 0,7\end{array}$ \\
\hline 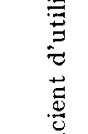 & Matières grasses & $\begin{array}{l}1 / 4-20 \\
23-3 t \\
37-48 \\
51-101\end{array}$ & $\begin{array}{l}89,8 \pm 6,2 \\
97,5 \pm 0,4 \\
97,0 \pm 0,8 \\
96,2 \pm 1,6\end{array}$ & $\begin{array}{l}76,5 \pm 6,5 \\
95,8 \pm 0,7 \\
96,6 \pm 0,8 \\
93,8 \pm 1,5\end{array}$ & $\begin{array}{l}69,0 \pm 5,5 \\
91,2 \pm 0,3 \\
92,0 \pm 2,5 \\
93,1 \pm 0,8\end{array}$ \\
\hline 8 & Matières minćrales & $\begin{array}{l}11_{4}-20 \\
23-34 \\
37-48 \\
51-101\end{array}$ & $\begin{array}{l}87,9 \pm 6,5 \\
95,2 \pm 0,5 \\
95,4 \pm 1,6 \\
93,2 \pm 2,0\end{array}$ & $\begin{array}{l}70,4 \pm 12,8 \\
89,9 \pm 1,2 \\
91,7 \pm 1,6 \\
90,8 \pm 1,7\end{array}$ & $\begin{array}{l}65,1 \pm 1,8 \\
89,4 \pm 1,0 \\
89,5 \pm 3,2 \\
91,4 \pm 0,7\end{array}$ \\
\hline Coeffici & $\begin{array}{l}\text { de rétention apparente } \\
\text { e l'azote }(\%)\end{array}$ & $1 / 4-101$ & $64,5 \pm 12,5$ & $63,9 \pm 10,6$ & $59,6 \pm 16,9$ \\
\hline $\begin{array}{l}\text { Quant } \\
\text { retenu/ }\end{array}$ & $\begin{array}{l}\text { ''azote apparemment } \\
\text { le gain de poids vif }(g)\end{array}$ & $1 /-101$ & 36,9 & 41,6 & 38,0 \\
\hline
\end{tabular}

L'utilisation digestive de la matière organique (tabl. 3) du lait témoin a été élevée dès la première période de mesure ( $94 \mathrm{p}$. I $\mathrm{Oo}$ ) ; elle a augmenté légèrement entre la première et la seconde période (97 p. Ioo) pour demeurer sensiblement constante par la suite ; cela correspond à l'évolution qui est généralement observée avec les aliments d'allaitement classiques riches en lait écrémé (Toullec et MAThieu, I969). L'utilisation digestive de la matière organique des laits citrate et acide a été moins élevée que celle du lait témoin au cours de la première période (respectivement 88 et 84 p. Ioo). L'écart observé entre le lait témoin et le lait citrate est devenu très faible dès la seconde période. Celui observé entre le lait témoin et le lait acide est devenu moins important à partir de la seconde période et a été très faible par la suite. L'utilisation digestive des matières azotées, des matières grasses et des matières minérales a suivi une évolution analogue à celle de la matière organique (tabl. 3), mais les différences observées au cours du 
premier mois ont été généralement plus prononcées, surtout entre le lait acide d'une part, et les laits témoin et citrate d'autre part; ainsi, au cours de la première période, l'utilisation digestive apparente des matières azotées a été respectivement de 75, 92 et $87 \mathrm{p}$. Ioo au lieu de 95, 96 et $95 \mathrm{p}$. 100 pendant les 5 dernières périodes. L'utilisation digestive du lait de remplacement n'a donc été fortement affectée que jusqu'à l'âge d'environ 3 semaines par la suppression partielle de la coagulation et jusqu'à l'âge d'environ un mois par sa suppression totale. L'absence de coagulation explique donc l'utilisation digestive moins élevée (de 5 à 16 p. Ioo pour la matière organique) que nous avons généralement constatée au cours du premier mois par rapport aux périodes suivantes, pour les laits de remplacement dépourvus de caséine (Toullec et al., I969; Paruelle. et al., 1972). En revanche, les différences observées au-delà du premier mois dans l'utilisation digestive, entre les protéines du lait et celles de remplacement ne seraient pas déterminées par l'absence de coagulation mais plutôt par l'origine des matières azotées et la technologie de leur préparation. Le système digestif du très jeune veau paraît donc insuffisamment adapté à l'utilisation des protéines ne formant pas un coagulum ferme dans la caillette. L'équipement enzymatique protéolytique pancréatique, en particulier, est encore peu développé (Ternouth, Siddons et Toothili, I97I ; Ternouth et Roy, 1973) et le rôle de la caillette dans la digestion pourrait alors être plus important que par la suite.

En conclusion la modification ou la suppression de la coagulation des protéines du lait par addition de citrate de sodium ou d'acide chlorhydrique n'a pas eu de conséquence néfaste sur la fréquence des diarrhées. L'utilisation digestive n'a été notablement diminuée que jusqu'à l'âge de trois semaines ou d'un mois. L'absence de coagulation ne semble donc pas constituer un obstacle au remplacement total des protéines du lait écrémé dans les aliments d'allaitement ; cependant, au cours du premier mois, il serait probablement utile de conserver une proportion importante de lait écrémé, pour maintenir une utilisation digestive élevée.

Rę̧u pour publication en avril 1974.

\section{SUMMARY}

\section{EFFECT OF COAGULATION OF MILK PRO'TEIN ON THE DIGESTIBILITY OF A MILK REPIACER IN PRERUMINANT CALVES}

The effect of protein coagulation on the digestibility of a milk replacer containing 67 p. Ioo skim-milk powder according to dry matter was studied. To this end, partial or complete uncoagulability of the milk was obtained by adding sodium citrate or hydrochloric acid at the moment of feeding. The milk replacers were offered to 3 preruminant calves kept in balance cages between ages of about 8 and roo days. Appetite, growth and feed efficiency were more favourable in the animals receiving control milk and citrate milk than in those fed the acid milk (table 2). Change or suppression of coagulation did not have any unfavourable effect on the frequency of diarrhoea. The digestibility (table 3) was only markedly reduced till the age of three weeks or one month. Thus, the apparent digestibility coefficients (ADC) of the organic matter were 94,88 and 84 p. Ioo respectively for control, citrate and acid milk replacers versus 97,97 and $96 \mathrm{p}$. roo from the age of one month. This finding confirms that the absence of coagulation does not constitute an obstacle to total replacement of milk protein in milk replacers. However, during the first month, it would probably be advisable to maintain a large proportion of skimmilk in the diet.

\section{RÉFÉRENCES BIBLIOGRAPHIQUES}

Blaxter K. L., WoOD W. A., 1953. Some observations on the biochemical and physiological events associated with diarrhoea in calves. Vet. Rec., 50, $889-892$. 
Burt A. W. A., IRvine S. M., 1972. Effect of deliberate dietary mismanagement upon the incidence of diarrhoea in homebred calves. Anim. Prod., 14, 299-308.

Frantzen J.-F., Toullec R., Thrvend P., Mathieu C.-M., I973. Influence de la coagulation des protéines sur la vidange stomacale chez le Veau préruminant. Ann. Biol, anim. Bioch. Biophys., 13, $718-72 \mathrm{I}$.

Kastelic L., Bentley G., Phillips P., r950. Studies on growth and survival of calves fed semisynthetic milk from birth. J. Dairy Sci., 33, 725-736.

Netke S. P., Gardner K. E., Kendall K. A., r962. Effect of diet pH on fecal consistency of young calves. J. Dairy Sci., 45, 105-108.

Owen F. G., Jacobson N. L., Allen R. S., Homeyer P. G., I958. Nutritional factors in calf diarrhoea. J. Dairy Sci., 41, 662-670.

Paruelle J.-L., Toullec R., Frantzen J.-F., Mathieu C.-M., I972. Utliisation des protéines par le Veau préruminant à l'engrais. I. Utilisation digestive des protéines de soja et des levures d'alcanes incorporées dans les aliments d'allaitement. Ann. Zootech., 21, 319-33r.

Ternouth J. H., Roy J. H. B., r973. The effect of diet and feeding technique on digestive function in the calf. Ann. Rech. Vet., 4, 19-30.

Ternouth J. H., Sidpons R. C., Toothill J., I97I. Pancreatic secretion in the milk fed calf. Proc. Nutr. Soc., 30, 89 A.

Toullec R., Mathieu C.-M., ig69. Utilisation digestive des matières grasses et de leurs principaux acides gras par le Veau préruminant à l'engrais. Influence sus la composition corporelle. Ann. Biol. anim. Bicch. Biophys., 9, I39-I60.

Toullec R., Mathieu C.-M., Vassal L., Pion R., ig69. Utilisation digestive des protéines du lactosérum par le Veau préruminant à l'engrais. Ann. Biol. anim. Bioch. Biophys., 9, 66r-664.

Toullec R., Thivend P., Mathiev C.-M., I97r. Utilisation des protéines du lactosérum par le Veau préruminant. I. Vidange stomacale comparée du lait entier et de deux laits de remplacement ne contenant que des protéines de lactosérum comme source de matières azotées. Ann. Biol. anim. Bioch. Biophys., 11, 435-453.

Toullec R., Patureau-Mirand P., Paruelle J.-L., Guilermet R., I973. Utilisation des protéines par le Veau préruminant à l'engrais. Bull. Soc. Scient. Hyg. Alim., 61, 57-92. 